\title{
Assessing Human Cortical Activation and Network During Pitch Discrimination Task in Quiet and in Noisy Background
}

\author{
A. N. Yusoff ${ }^{1}$, K. Abdul Hamid ${ }^{1}$, M. Mohamad ${ }^{1}$, A. Abdullah' ${ }^{2}$, H. Abdul Hamid ${ }^{3}$ \& S. Z. M. Mukari ${ }^{4}$ \\ ${ }^{1}$ Diagnostic Imaging \& Radiotherapy Program, School of Diagnostic and Applied Health Sciences, Faculty of \\ Health Sciences, Universiti Kebangsaan Malaysia, Jalan Raja Muda Abdul Aziz, Kuala Lumpur, Malaysia \\ ${ }^{2}$ Department of Otorhinolaryngology, Faculty of Medicine, Universiti Kebangsaan Malaysia Medical Centre, \\ Jalan Yaacob Latif, Bandar Tun Razak, Cheras, Kuala Lumpur, Malaysia \\ ${ }^{3}$ Department of Radiology, Faculty of Medicine, Universiti Kebangsaan Malaysia Medical Centre, Jalan Yaacob \\ Latif, Bandar Tun Razak, Cheras, Kuala Lumpur, Malaysia \\ ${ }^{4}$ Audiology Program, School of Rehabilitation Sciences, Faculty of Health Sciences, Universiti Kebangsaan \\ Malaysia, Jalan Raja Muda Abdul Aziz, Kuala Lumpur, Malaysia \\ Correspondence: A. N. Yusoff, Diagnostic Imaging \& Radiotherapy Program, School of Diagnostic and Applied \\ Health Sciences, Faculty of Health Sciences, Universiti Kebangsaan Malaysia, Jalan Raja Muda Abdul Aziz, \\ Kuala Lumpur 50300, Malaysia. Tel: 6-3-2687-8084. E-mail: nazlim@fsk.ukm.my
}

Received: August 14, 2013

doi:10.5539/mas.v7n10p42
Accepted: September 12, $2013 \quad$ Online Published: September 24, 2013

URL: http://dx.doi.org/10.5539/mas.v7n10p42

\begin{abstract}
In spite of extensive research conducted in studying pitch memory processing, knowledge about the functional anatomy of the brain while performing pitch discrimination task is still lacking. The purpose of this study was to investigate the effects of background noise and tonal frequencies on brain activation during a pitch discrimination task. Thirteen participants were presented with categories of low- and high-frequency tones during an fMRI scan. They listen, recognize and discriminate the target tone with the last tone in a series of four distracting tones. Cortical responses during which the participants engaged with the pitch discrimination task were explored globally and differentially with tonal frequency and background condition as factors. Similar integrative network consisting of bilateral superior temporal gyrus, pre-central gyrus, cerebellum, insula, putamen, inferior parietal lobe and supplementary motor area is established during the four pitch discrimination task conditions. The bilateral A1 and right M1 are effectively activated by noise and action respectively. Background noise increases the participants' performance hence equalizing that in quiet, while participants performed significantly better in discriminating low-frequency tones as compared to high-frequency tones. This study revealed an integrative network that consists of areas responsible for pitch discrimination strategy. The bilateral primary auditory cortex played important roles in increasing participants' pitch discrimination performance in noisy surrounding and in discriminating low frequency category of tones.
\end{abstract}

Keywords: pitch memory, fMRI, white noise, auditory cortex, SPM, pitch discrimination

\section{Introduction}

Pitch memory is the ability of a person to recall a previously sounded tone. In an early pitch memory experiment (Czachowska-Sieszycka \& Budohoska, 1979), participants were required to listen to a tone (test tone 1) followed by a sequence of distracting tones and test tone 2 . They were then required to make a decision whether test tone 2 is similar or different to test tone 1 given earlier. From the results, the researchers concluded that the time intervals between test tones 1 and 2 as well as between the distracting tones have crucial influence on pitch memory. There were also several earlier studies (Deutsch, 1970, 1972a, 1972b, 1978) applying different non-imaging techniques in order to explore how human brain perceives and recognizes pure tones. In one of the studies, recognition of a pitch of a tone was found to be severely disrupted by the incorporation of intermediate tones between the two tones that need to be discriminated (Deutsch, 1970). Further studies by the same author indicated that pitch memory involves a storage which is arranged logarithmically in a highly ordered and specific fashion (Deutsch, 1972b). It was also found that errors in discriminating any two tones very much depend on whether the frequency of the two tones is similar or not and whether their frequencies are similar or not to the 
distracting tones delivered during the retention period (Deutsch, 1972a). In a latter communication, left-hand dominant participants have been found to perform pitch discrimination task significantly better than right-hand dominant participants (Deutsch, 1978).

There is a fundamental difference between pitch matching and pitch discrimination. While the earlier is defined by being able to vocally duplicate the pitch of a tone, the latter is understood as the ability to make an accurate judgment (similar or different) about two tones (Moore et al., 2008; Moore et al., 2007). Pitch matching and pitch discrimination have been found to be significantly correlated to each other in the absence of memory interference (Moore et al., 2007) when tones are used. However, their relationship becomes insignificant due to the reduction of pitch discrimination ability with the inclusion of intervening tones between the two tones that need to be discriminated (Moore et al., 2007). In a later study by the same author, no significant correlation between pitch matching and pitch discrimination was found when the stimuli used were participant's own voice, a natural female voice and non vocal complex tones (Moore et al., 2008). Nevertheless, the brain electrophysiological findings obtained from a pitch discrimination study across a frequency range of 250-4000 $\mathrm{Hz}$ have been found to correlate with the corresponding behavioral study (Novitski et al., 2004). The authors measured mismatch negativity (MMN) amplitude and latency, P3a amplitude and latency, participants' performance in answering and participants' reaction time in studying frequency discrimination performance at different frequency levels $(250-4000 \mathrm{~Hz})$. They found a transition point at $1000 \mathrm{~Hz}$ between two different neuronal mechanisms of pitch discrimination. They also found the existence of correlation between event-related potential (ERP) parameters (latencies and amplitudes) and behavioral test parameters such as percentage of correct answers and reaction time.

Even though there is a vast number of published work on pitch memory by researchers as mentioned above, most of the studies relied on the participants' performance for the interpretation of results without being able to project inside the human brain for additional information due to the lack of suitable imaging technology. Thus, many aspects of the functional anatomy of pitch memory was not understood, until the introduction of blood oxygenation level dependent (BOLD) (Ogawa et al., 1990) and positron emission tomography (PET) (Friston et al., 1991). These imaging techniques can be used to study brain function. Moreover, no background interferences were included in the previous studies to search for any significant effects on pitch memory performance. To our knowledge, the difference in brain responses to low frequency tones as compared to high frequency tones during a pitch discrimination task with background interference has yet to be completely studied using fMRI technique. Furthermore, there are differences in experimental methods and tasks used by previous workers that results in a wide range of experimental findings with regards to pitch memory such as the effect of the frequency of the pure tone on brain activation and the influence of time interval between and during the delivery of the tones on participants' performance. Thus, a direct comparison between the results obtained is seldom possible requiring more work to be conducted on this subject matter.

In this work, the effects of tonal frequency and background noise on cortical activation and the corresponding network during the performance of a pitch discrimination task were investigated. The motivation is based on the foundation that the underlying activation and network that ensues during the execution of pitch memory has not been fully explored especially when the task is performed using low- and high-frequency category of tones which are distracted by the background noise. The data were analyzed using Statistical Parametric Mapping (SPM8) (Functional Imaging Laboratory, Wellcome Department of Imaging Neuroscience, Institute of Neurology, University College of London) from which the spatial and height extent of activation in the brain were generated and then compared and contrasted i) between the execution of pitch discrimination task in quiet and in a noisy background and ii) between the execution of pitch discrimination task that used low- and high-frequency tones. Three research questions were imposed for this study which are i) does the brain uses the same cortical network when discriminating (involving listening, attention, recognizing and action) tones in quiet and in noise, ii) does tonal frequency and background noise influence the pitch discrimination performance and iii) is there any difference in brain activation when the pitch discrimination task is performed in noise as compared to in quiet (vice-versa) or using low- as compared to high-frequency tones (vice-versa). The hypotheses to be tested are anatomically constrained; i) if the same cortical mechanism and network are used by the brain in discriminating the tones in quiet and in noise, we should see similar activated areas as obtained by Gaab et al. (2003) and Gaab and Schlaug (2003) for all the experimental conditions used in this study and ii) if noise and tonal frequency does influence participants' performance, we should see some significant activations in the cortical auditory areas in the brain. 


\section{Methods}

\subsection{Participants}

Thirteen healthy right-handed Malay participants $(7$ males) agreed to participate by filling up the informed consent and screening forms and signing them, after being given full explanation of the nature and risks of the research, as required by the Institutional Ethics Committee (IEC) (Reference no: NN-197-2010). The participants were then interviewed on their health condition prior to the scanning session and were tested for handedness using Edinburgh Handedness Inventory (Oldfield, 1971). The participants were also asked whether they have received any musical education or training. All participants were tested for their middle ear condition and hearing level using a tympanometer (Model Grason Stadler Inc. GSI33) and a pure-tone audiometer (Model Grason Stadler Inc. GSI61) respectively, by a qualified Audiologist. Pure tone audiometry was conducted in the frequency range of $250 \mathrm{~Hz}$ to $8000 \mathrm{~Hz}$.

\section{2 fMRI Scans}

A sparse temporal sampling functional magnetic resonance imaging (STS-fMRI) was used in this study (Figure 1) in order to avoid the interference of the scanner sound with the auditory paradigm. STS-fMRI is the technique of choice in auditory studies due to its relatively high sensitivity in detecting brain activation (Mueller et al., 2011). There were altogether 218 functional scans in every imaging session. The first two scans were dummies (not shown in Figure 1) and were automatically discarded by the BOLD imaging protocol to eliminate the magnetic saturation effect. Each functional volume consisted of 35 axial slices that were acquired in 3-s acquisition time (TA) (one image slice in $85.7 \mathrm{~ms}$ ) with an inter-scan interval (TR) of $13 \mathrm{~s}$. The imaging time for the whole functional scan is $2808 \mathrm{~s}$ (46.8 minutes) which produces $216 \times 35=7560$ images in total. The participants were told not to move their head during the scan and were instructed to attend and respond to the given stimulus accordingly.

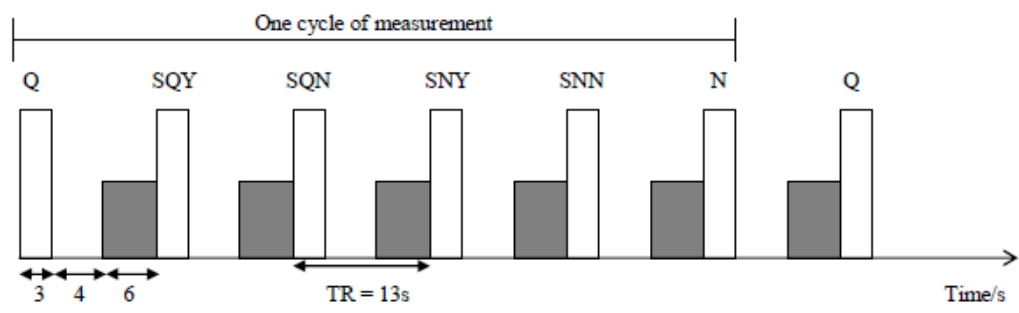

(a)

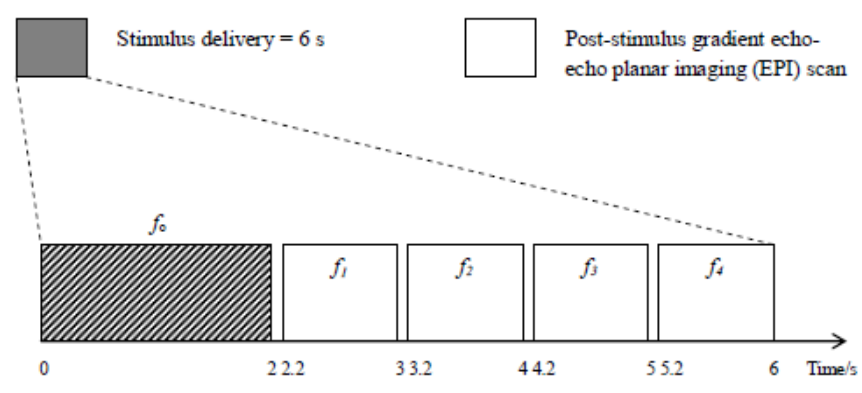

Pure tone with target frequency $\left(f_{0}\right)$

Pure tones of different frequencies $\left(f_{1}, f_{2}, f_{3}, f_{4}\right)$ to be discriminated with $f_{0}$

(b)

Figure 1. Schematic representation of (a) timing diagram and stimulus delivery and (b) the details of the stimulus delivery part shown in (a)

The above mentioned STS-fMRI scans were performed using a 3-tesla magnetic resonance imaging (MRI) system (Siemens Magnetom Verio) equipped with functional imaging option, echo planar imaging capabilities and radiofrequency (RF) head coil used for signal transmission and reception. Gradient Echo-Echo Planar Imaging (GRE-EPI) pulse sequence with the following parameters were applied to produce high resolution 
T2*-weighted images: Repetition time $(\mathrm{TR})=13000 \mathrm{~ms}$, acquisition time $(\mathrm{TA})=3000 \mathrm{~ms}$, interscan interval $(\mathrm{TA})=13000 \mathrm{~ms}-3000 \mathrm{~ms}=10000 \mathrm{~ms}$, echo time $(\mathrm{TE})=50 \mathrm{~ms}$, field of view $(\mathrm{FOV})=192 \times 192 \mathrm{~mm}$, flip angle $(\alpha)=90^{\circ}$, voxel size $=3 \mathrm{~mm}^{3}$ isotropic, slice thickness $=3 \mathrm{~mm}$. In addition, anatomical images of the entire brain were obtained using a T1-weighted multiplanar reconstruction (MPR) pulse sequence with the following parameters: $\mathrm{TR}=1620 \mathrm{~ms}, \mathrm{FOV}=250 \times 250 \mathrm{~mm}, \alpha=90^{\circ}$, voxel size $=3 \mathrm{~mm}^{3}$ isotropic and slice thickness $=3 \mathrm{~mm}$.

\subsection{Pitch Discrimination Task}

As can be seen in Figure 1, the STS-fMRI post-stimulus scans were carried out following the delivery of series of pure tones that were generated using Adobe Audition 2.0 software (Adobe Systems Inc., San Jose, CA, USA). The tones were alternatively delivered in quiet $(\mathrm{Q})$ and in noisy backgrounds $(\mathrm{N})$. A broad-band white noise stimulus was chosen to be used as background in this study due to its well known effectiveness in evoking brain responses especially in the temporal lobe region (Langers et al., 2005). Thus, the brain activation obtained solely from listening to white noise is used as the activation landmark in this study. Six pure tones with frequencies of $700 \mathrm{~Hz}, 1000 \mathrm{~Hz}$ and $2000 \mathrm{~Hz}$, categorized as low-frequency tones and $3500 \mathrm{~Hz}, 4000 \mathrm{~Hz}$, and $4500 \mathrm{~Hz}$ categorized as high frequency tones were used. All the pure tone stimuli were recorded and presented at equal amplitudes. The schematic representation of the timing paradigm and stimulus delivery are illustrated in Figure 1 (a) and (b) and will be explained in the following paragraph.

Each STS-fMRI session has 36 trials and each trial consisted of six conditions. The six conditions that contained a trial are shown in Figure 1(a) which are i) quiet condition $(\mathrm{Q})$ in which the participants were instructed to stay calm, ii) stimulus given in a quiet background (SQY) in which the participants were supposed to respond, iii) stimulus given in a quiet background (SQN) in which the participants were not supposed to respond, iv) stimulus given in a noisy background (SNY) in which the participants were supposed to respond, v) stimulus given in a noisy background (SNN) in which the participants were not supposed to respond and finally vi) listening only to white noise stimulus $(\mathrm{N})$.

As shown in Figure 1(b), the stimulus which is a series of pure tones named as the target tone $\left(f_{0}\right)$ and the distracting tones $\left(f_{1}, f_{2}, f_{3}\right.$ and $\left.f_{4}\right)$ are grouped together in either high or low frequency category. Due to the inavailability of the electrostatic headphone on our 3T-system, the stimulus was binaurally delivered to the participants via an MRI-compatible headphone connected to a digital audio playback system through air-conducting tubes. The intensity level of the stimulus is kept constant at $70 \mathrm{~dB}$ higher than normal human sound pressure level (SPL). The white noise, pure tones in quiet background and pure tones in noisy background are satisfactorily received and heard, as claimed by the participants. The duration of the stimulus from the onset of $f_{0}$ until the end of $f_{4}$ is 6 seconds. The duration of $f_{0}$ is $2 \mathrm{~s}$ while each of the distracting tone lasts $0.8 \mathrm{~s}$. The gap between consecutive tones is kept at $0.2 \mathrm{~s}$. The participants were instructed to pay attention, recognize, discriminate and match the target tone $\left(f_{0}\right)$ with the last tone $\left(f_{4}\right)$ of the four distracting tones. If the participants notice that both the $f_{0}$ and $f_{4}$ are similar, they were required to press the squeeze bulb using his/her left hand but remain calm if otherwise. The participants' response were recorded manually outside the MR examination room and were later compared with the correct answers to determine their behavior performance. To minimize habituation effects, the frequency values for the tone sequence $f_{0}, f_{1}, f_{2}, f_{3}, f_{4}$ are randomized and the low- and high-frequency 6-s stimuli were also randomly given during SQY, SQN, SNY and SNN conditions. For SNY and SNN conditions, the stimulus-to-noise ratio (SNR) was fixed at $-5 \mathrm{~dB}$.

\subsection{Image Processing}

The fMRI data were analysed using MATLAB 7.4-R2008a (Mathworks Inc. MA, USA) and Statistical Parametric Mapping (SPM8) programming softwares. Functional images in each measurement were realigned using the 6-parameter affine transformation in translational ( $x, y$ and $z$ ) and rotational (pitch, roll and yaw) directions to reduce artifacts from participant's movements. After realigning the data, a mean image of the series is used to estimate some warping parameters that map it onto a template that already conforms to a standard anatomical space (EPI template provided by the Montreal Neurological Institute-MNI). The normalization procedure used a 12-parameter affine transformation. The images were then smoothed using an 8-mm full-width-at-half-maximum (FWHM) Gaussian kernel. Low-frequency responses caused by aliased biorhythms, cardiac effects and other oscillatory signal variations were removed using high-passed filter. Conventional group fixed-effects (FFX), random-effects (RFX) and conjunction analyses (Friston et al., 1999) based on the general linear model (GLM) (Friston, 2005) were used to generate brain activation. The designed matrix was uniquely created according to the participants' answer that was recorded at the control panel outside the MRI room. For example, the column "SQY" in the designed matrix contains only measurements that the participants answered 
"YES" and "PRESS" the squeeze bulb when the pitch discriminating task was conducted in "QUIET" background but their answers were not necessarily "TRUE" according to the answer scheme prepared by the researchers. Similarly, the column "SNN" contains measurements that the participants "DID NOT PRESS" the squeeze bulb when the pitch discriminating task was conducted in "NOISY" background but their answers were not necessarily "TRUE" according to the answer scheme prepared by the researchers. The same goes for "SQN" and "SNY" columns in the designed matrix.

In FFX and conjunction analyses, the activations were height-thresholded at a corrected $\alpha=0.05$ to control the family-wise error. In RFX analysis, the activations were height-thresholded at uncorrected significant level $(\alpha)=$ 0.001 with a spatial threshold ( $k$ ) of 100 voxels (Friston et al., 1996; Stoessel et al., 2011). These cut-offs in RFX framework mean that any voxel with a $t_{\mathrm{df}}(\mathrm{df}=$ degree of freedom) value larger than $3.646(p<0.001)$ that belongs to a cluster of not less than 100 voxels is considered significantly activated (Stoessel et al., 2011). The $t_{\mathrm{df}}$ and $p$ values, number of activated voxel in a cluster of activation, the MNI (Montreal Neurological Institute) coordinates and the respective areas obtained from the group FFX, conjunction and RFX activation maps were recorded for evaluation. The cortical regions which were found to be significantly activated in all the analyses were evaluated using the Anatomy toolbox for SPM (Eickhoff et al., 2005).

Differential brain activation due to the effects of noise and action was studied using paired $t$ tests and differential method implemented in FFX and RFX frameworks. This is done in order to validate the experimental fMRI paradigm used in this study. Differential brain activation due to the effects of performing the tone discrimination task in quiet and in noisy background using i) low and ii) high frequency tones was also studied using paired $t$ tests. Similar tests were conducted to search for the difference in brain activation when the tone discrimination task is performed using low and high frequency tones in i) quiet and ii) noisy background. This analysis also used a unique designed matrix in which fMRI measurements are separated in columns of the designed matrix according to the backgrounds which are in quiet or in noise (regardless of the tonal frequency used in the pitch discrimination task) and according to the tonal frequency which are low or high frequencies (regardless of the background of the pitch discrimination stimulus). All results obtained from differential brain activation studies are reported at uncorrected significant level $(\alpha)=0.001$ with a spatial threshold of 100 voxels.

\subsection{Behavioral Study}

The participant's performance in the frequency discriminating task in quiet and in noise using low and high frequency tones was recorded outside the scanner and was scored as the number of correct match between $f_{\mathrm{o}}$ and $f_{4}$. Qualitative and quantitative interpretations on the participant's performance are made in relation to the fMRI results.

\section{Results}

\subsection{Demographical Results}

The participants' average age and standard deviation is $27.2 \pm 4.5$ years. All the thirteen participants were tested to be strongly right-handed with average laterality index $\left(\mathrm{LI}_{\mathrm{ave}}\right)$ of 85.8 , in the range of $6^{\text {th }}$ to $7^{\text {th }}$ right, as assessed using Edinburgh Handedness Inventory (Oldfield, 1971). All participants reported no history of psychiatric or neurological disorder and no current use of any psychoactive medications. None of the participants were professional musician and none of the participants actively played any musical instruments. The participants had no hearing impairment and no history of long time exposure to loud noise to be inappropriate for auditory stimulus presentation. The participants' hearing levels for both ears are not greater than $30 \mathrm{~dB}(\mathrm{HL})$ in the frequency range of $250-8000 \mathrm{~Hz}$.

\subsection{Brain Activations Due to Listening to Noise and During Pitch Discrimination Task}

Listening only to noise ( $N$ condition) activated Heschl's gyrus (HG) bilaterally with a higher $t$ value and number of activated voxels (NAV) for the right HG. The RFX results at uncorrected significant level $(\alpha)=0.001$ and extent threshold $(k)=100$ voxels indicate a number of 270 voxels that were activated in the right HG (TE1.0, TE1.1 and TE1.2) as compared to 107 voxels in the left HG (TE1.0, TE1.1 and TE1.2). Activation peaks are at $+46 /-18 /+8\left(t_{12}=5.49\right)$ and $-46 /-14 /+4\left(t_{12}=5.34\right)$ in the right and left hemispheres respectively. The RFX results are in good agreement with that obtained from FFX at corrected significance level $(\alpha)=0.05$ and extent threshold $(k)=100$ voxels which indicates a number of 568 voxels that were activated in the right HG (TE1.0 and TE1.1) as compared to 230 voxels in the left HG (TE1.0 and TE1.1). Activation peaks are at $+48 /-14 /+4$ $\left(t_{2093}=8.48\right)$ and $-40 /-24 /+6\left(t_{2093}=7.36\right)$ in the right and left hemispheres respectively. Both the RFX and FFX results point to the right lateralization (right more than left) of the primary auditory cortex activation when listening to white noise. 
Performing each of the pitch discrimination tasks which are SQY, SQN, SNY and SNN resulted in a similar widespread of activation in the temporal, parietal and frontal lobes as well as in the cerebellum. Thus, to illustrate the common activated areas for all the SQY, SQN, SNY and SNN tasks, the brain activation, as shown in Figure 2, were obtained from the analysis of conjunction at the first level (FFX) analysis. The activation was thresholded at corrected significance level $(\alpha)=0.05$. Common activation areas for all the four pitch discrimination conditions can be observed, from top to bottom rows, in the cerebellar vermis and cerebellum (VI) $\left(1^{\text {st }}\right.$ and $2^{\text {nd }}$ rows $)$, inferior parietal lobes $\left(2^{\text {nd }}\right.$ and $3^{\text {rd }}$ rows $)$, superior temporal gyrus $\left(3^{\text {rd }}\right.$ and $4^{\text {th }}$ rows $)$, precentral gyrus $\left(5^{\text {th }}\right.$ row $)$, supplementary motor area $\left(5^{\text {th }}\right.$ and $6^{\text {th }}$ rows $)$, putamen $\left(5^{\text {th }}\right.$ row $)$, insula lobe $\left(6^{\text {th }}\right.$ row $)$ and inferior frontal gyrus $\left(6^{\text {th }}\right.$ row). It was found that all the statistical maps for SQY, SQN, SNY, SNN and conjunction analysis exhibited SMA as the region of highest intensity. Statistical details about the brain activation shown in Figure 2 are given in Table 1. It can be clearly seen that bilateral activation occurs in the cerebellum, supplementary motor area, superior temporal gyrus, precentral gyrus, middle temporal gyrus, inferior parietal lobe, insula and putamen. However, the inferior frontal gyrus is fully right lateralized. From Table 1, it can also be observed that the regions with the four highest $t_{\mathrm{df}}$ values $\left(t_{2093}>10.00, p_{\text {cluster }}<0.001, p_{\text {voxel }}<0.001\right)$ are bilateral supplementary motor area, superior temporal gyrus, cerebellum (VI) and pre-central gyrus with right lateralised height extent of activation - right higher than left. For inferior parietal lobe, insula and putamen that also show bilateral activation, the right hemisphere areas are always higher in the $t$ value and number of activated voxel as compared to their counterpart in the left hemisphere.

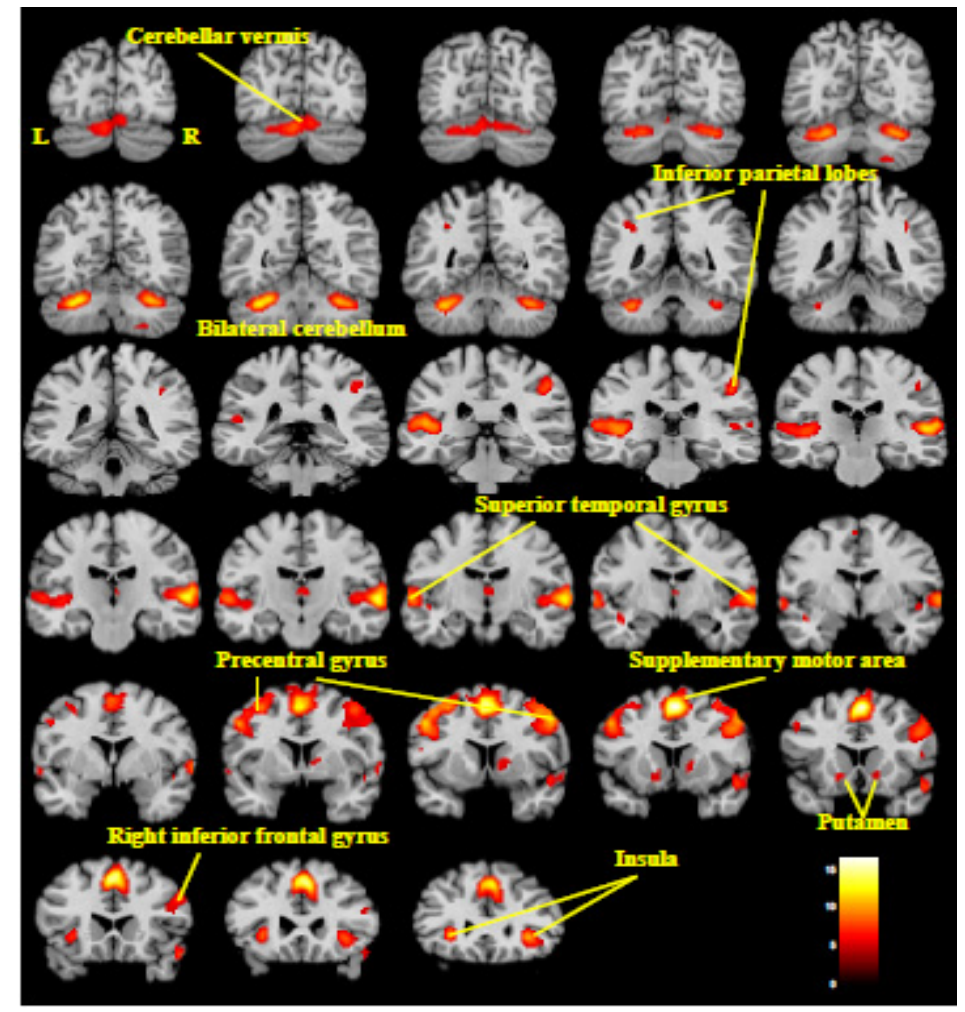

Figure 2. Brain activation in coronal orientation obtained from conjunction analysis of the four experimental conditions for pitch discrimination task across all participants overlaid onto colin27T1_seg.img T1 weighted template

\subsection{Validation of the Pitch Discrimination Task: The Significant Effects of Noise and Action}

Paired $t$-test was done in the second level analysis to obtain the significant effects of white noise during the performance of pitch discrimination task. In the method, the significant effects of noise was obtained by combining the contrast images of SNY and SNN for every participants and subtracting the average effects with the average effects of the combination of contrast images of SQY and SQN contrast images for all participants. The activated voxels obtained are the voxels that are significantly more active in noise as compared to in quiet regardless of whether the participant pressed or did not press the squeeze bulb. The activation was thresholded at $p_{\text {uncorr }}=0.001$ and $k=100$ voxels. Two significantly activated clusters in the right and left temporal lobes 
survived the threshold, see Table 2(a). Specific areas of activation are the right Heschl's and superior temporal gyrus which are assigned to right TE1.0, TE.1.1 and TE1.2 and left superior temporal gyrus and left Rolandic Operculum assigned to left TE1.1, TE1.2 and OP4. However, only a small difference in the $t_{\mathrm{df}}$ values (for the two local maxima) and the number of active voxels were observed for those two left and right hemispheric clusters.

Due to the expectation that listening intentionally and unintentionally to noise should result in different brain activation, similar differential brain activation analysis was then performed in the FFX framework. The results at corrected significant level $(\alpha)=0.05$ and extent threshold $(k)=0$ voxels indicate a number of 849 voxels that were activated in the right auditory area, assigned to right Heschl's gyrus (TE1.0, TE1.1) and right superior temporal gyrus (TE1.0) as compared to 1001 voxels in the left auditory area, assigned to left superior temporal gyrus (TE1.0, TE1.1 and TE1.2), see Table 2(b). Activation peaks are at $+46 /-18 /+8\left(t_{2093}=8.93\right)$ and $-42 /-24 /+8$ $\left(t_{2093}=10.41\right)$ in the right and left hemispheres respectively.

Table 1. Significant activation clusters obtained from the conjunction of SQY, SQN, SNY and SNN conditions across all participants at corrected $\alpha=0.05$. A maximum of three local maxima more than $8.0 \mathrm{~mm}$ apart are shown

\begin{tabular}{|c|c|c|c|c|c|c|}
\hline $\begin{array}{l}\text { Activation } \\
\text { cluster }\end{array}$ & $p_{\text {cluster }}$ & $N A V$ & $p_{\text {voxel }}$ & $t_{2093}$ & $x / y / z$ & $\begin{array}{l}\text { Activation } \\
\text { areas }\end{array}$ \\
\hline \multirow[t]{3}{*}{1} & $*$ & 2063 & $*$ & 16.40 & $+2 /+10 /+58$ & Right supplementary motor area \\
\hline & & & $*$ & 15.00 & $0 /+18 /+52$ & Left supplementary motor area \\
\hline & & & 0.001 & 5.47 & $-4 /+2 /+76$ & Left supplementary motor area \\
\hline \multirow[t]{3}{*}{2} & $*$ & 1455 & $*$ & 13.51 & $+62 /-20 /+4$ & Right superior temporal gyrus \\
\hline & & & $*$ & 7.91 & $+52 /+12 /-14$ & Right temporal pole \\
\hline & & & $*$ & 6.58 & $+46 /-16 /+2$ & Right superior temporal gyrus \\
\hline \multirow[t]{3}{*}{3} & $*$ & 2185 & $*$ & 12.83 & $-32 /-62 /-28$ & Left cerebellum (VI) \\
\hline & & & $*$ & 11.00 & $+30 /-62 /-26$ & Right cerebellum (VI) \\
\hline & & & $*$ & 8.36 & $-8 /-80 /-20$ & Left cerebellum (Crus 1) \\
\hline \multirow[t]{3}{*}{4} & $*$ & 811 & $*$ & 11.29 & $-50 /+4 /+42$ & Left precentral gyrus \\
\hline & & & $*$ & 9.81 & $-32 /+2 /+58$ & Left middle temporal gyrus \\
\hline & & & $*$ & 9.69 & $-44 /+4 /+54$ & Left precentral gyrus \\
\hline \multirow[t]{3}{*}{5} & * & 946 & $*$ & 10.99 & $+50 /+4 /+46$ & Right precentral gyrus \\
\hline & & & $*$ & 9.73 & $+42 /+2 /+54$ & Right middle temporal gyrus \\
\hline & & & $*$ & 9.05 & $+48 /+8 /+38$ & Right precentral gyrus \\
\hline \multirow[t]{3}{*}{6} & $*$ & 1232 & $*$ & 10.21 & $-48 /-36 /+12$ & Left superior temporal gyrus \\
\hline & & & $*$ & 9.32 & $-60 /-18 /+4$ & Left superior temporal gyrus \\
\hline & & & $*$ & 8.84 & $-60 /-34 / 12$ & Left superior temporal gyrus \\
\hline 7 & $*$ & 356 & $*$ & 9.87 & $+32 /+26 /-2$ & Right insula lobe \\
\hline 8 & $*$ & 215 & $*$ & 8.91 & $-30 /+24 /+2$ & Left insula lobe \\
\hline \multirow[t]{2}{*}{9} & * & 240 & $*$ & 8.11 & $+46 /-34 /+46$ & Right inferior parietal lobe \\
\hline & & & 0.02 & 5.40 & $+40 /-46 /+44$ & Right inferior parietal lobe \\
\hline 10 & $*$ & 110 & $*$ & 6.79 & $+46 /+36 /+24$ & Right inferior frontal gyrus \\
\hline 11 & 0.001 & 41 & $*$ & 6.18 & $+24 /-66 /-50$ & Right cerebellum (VIII) \\
\hline 12 & 0.001 & 44 & $*$ & 5.85 & $-14 /+10 /-8$ & Left putamen \\
\hline \multirow[t]{2}{*}{13} & $*$ & 109 & 0.001 & 5.62 & $+12 /+2 /+8$ & Right putamen \\
\hline & & & 0.003 & 5.26 & $+14 /+12 /-6$ & Right putamen \\
\hline 14 & 0.001 & 49 & 0.001 & 5.52 & $-36 /-52 /+42$ & Left inferior parietal lobe \\
\hline 15 & 0.012 & 9 & 0.007 & 5.10 & $+52 /+12 /+12$ & Right inferior frontal gyrus \\
\hline 16 & 0.015 & 7 & 0.015 & 4.94 & $-54 /+6 /+20$ & Left precentral gyrus \\
\hline
\end{tabular}

$* p<0.001 ; N A V=$ number of activated voxels. 
Comparing the two FFX results, which are for listening intentionally to noise with that of differential brain activation analysis mentioned above (listening unintentionally), it can be said that a laterality shift has occured from right hemisphere during listening to noise alone to left hemisphere during the performance of pitch discrimination task in noise from which the local maximum $t_{2093}$ value has increases dramatically from 7.36 to 10.41 while the number of active voxels increases markedly from 230 to 1001 . The effects (which are not observable in RFX framework) could possibly due to the change from attentive listening to noise to inattentive listening to noise but with attentive listening to the pitch discrimination stimulus given in noisy background.

As mentioned in the method section, the participants need to press the squeeze bulb using their left hand whenever they would be able to discriminate between the target tone $\left(f_{\mathrm{o}}\right)$ and the last tone $\left(f_{4}\right)$ of the four distracting tones (e.g. upon noticing that $f_{\mathrm{o}}=f_{4}$ ). The effects of participants' action when attending to the given stimuli can also be obtained via a paired $t$-test. This is done by combining the contrast images of SQY and SNY for every partis and subtracting the average effects with the average effects of the combination of SQN and SNN contrast images of all participants. The activated voxels obtained are the voxels that are significantly more active when pressing the squeeze bulb as compared to not pressing regardless of whether the pitch discrimination task was performed in quiet or in noise. The activation was thresholded at $p_{\text {uncorr. }}=0.001$ with $k=100$ voxels. Only a single cluster of activation survived the threshold. This activation occurs in contralateral motor areas specifically in the right precentral gyrus involving BA6 and BA3a according to Brodmann classification (Table 2(a)). Similar activation (507 voxels) in the right precentral gyrus (BA6) contralateral to the hand used to press the squeeze bulb was obtained when the analysis was done in the FFX framework (Table 2(b)). Interestingly, FFX analysis revealed small ipsilateral activation (42 voxels) in the left post-central gyrus (BA6).

Table 2. Differential brain activation characteristics obtained from paired $t$-test in the (a) second and (b) first level analyses to obtain pure effects of noise and action

(a)

\begin{tabular}{|c|c|c|c|c|c|c|c|c|}
\hline \multirow{2}{*}{$\begin{array}{l}\text { No. of } \\
\text { active } \\
\text { cluster }\end{array}$} & \multirow{2}{*}{$\begin{array}{c}\text { Cluster } \\
\text { level } \\
p \text {-value }\end{array}$} & \multirow{2}{*}{$\begin{array}{l}\text { No. of } \\
\text { active } \\
\text { voxels }\end{array}$} & \multirow{2}{*}{$\begin{array}{c}\text { Peak } \\
\text { level } \\
p \text {-value }\end{array}$} & \multirow{2}{*}{$\begin{array}{c}\text { Peak } \\
\text { level } t_{12} \\
\text { value }\end{array}$} & \multicolumn{3}{|c|}{ MNI coordinates } & \multirow[t]{2}{*}{ Activated areas } \\
\hline & & & & & $x$ & $y$ & $z$ & \\
\hline \multicolumn{9}{|c|}{ Effects of noise $\left(p_{\text {uncorr. }}=0.001\right.$, extent threshold $=100$ voxels $)$} \\
\hline \multirow[t]{6}{*}{2} & $*$ & 755 & $*$ & 6.91 & 44 & -20 & 8 & Right Heschl's gyrus (TE1.0, TE1.1) \\
\hline & & & $*$ & 6.31 & 50 & -10 & -6 & Right superior temporal gyrus (TE1.0) \\
\hline & & & * & 6.26 & 54 & -14 & 4 & Right superior temporal gyrus (TE1.0, TE1.2) \\
\hline & $*$ & 707 & * & 6.7 & -40 & -26 & 2 & TE1.1 \\
\hline & & & * & 6.4 & -50 & -4 & -2 & Left superior temporal gyrus (TE1.2, OP4) \\
\hline & & & * & 4.57 & -36 & -34 & 16 & Left Rolandic Operculum (TE1.1) \\
\hline \multicolumn{9}{|c|}{ Effects of action $\left(p_{\text {uncorr. }}=0.001\right.$, extent threshold $=100$ voxels $)$} \\
\hline \multirow[t]{3}{*}{1} & 0.001 & 334 & $*$ & 4.68 & 40 & -18 & 58 & Right precentral gyrus (BA6, BA3a) \\
\hline & & & * & 4.17 & 32 & -16 & 52 & Right precentral gyrus (BA6) \\
\hline & & & * & 3.83 & 34 & -12 & 48 & Right precentral gyrus (BA6) \\
\hline
\end{tabular}

$* p<0.001$. 
(b)

\begin{tabular}{|c|c|c|c|c|c|c|c|c|}
\hline \multirow{2}{*}{$\begin{array}{l}\text { No. of } \\
\text { active } \\
\text { cluster }\end{array}$} & \multirow{2}{*}{$\begin{array}{c}\text { Cluster } \\
\text { level } \\
p \text {-value }\end{array}$} & \multirow{2}{*}{$\begin{array}{l}\text { No. of } \\
\text { active } \\
\text { voxels }\end{array}$} & \multirow{2}{*}{$\begin{array}{c}\text { Peak } \\
\text { level } \\
p \text {-value }\end{array}$} & \multirow{2}{*}{$\begin{array}{l}\text { Peak level } \\
t_{2093} \text { value }\end{array}$} & \multicolumn{3}{|c|}{ MNI coordinates } & \multirow[t]{2}{*}{ Activated areas } \\
\hline & & & & & $x$ & $y$ & $z$ & \\
\hline \multicolumn{9}{|c|}{ Effects of noise ( $p_{\text {corr. }}=0.05$, extent threshold $=100$ voxels) } \\
\hline \multirow[t]{4}{*}{2} & * & 1001 & $*$ & 10.41 & -42 & -24 & 8 & Left superior temporal gyrus (TE1.0, TE1.1) \\
\hline & & & * & 7.74 & -52 & -12 & -4 & Left superior temporal gyrus (TE1.2) \\
\hline & * & 849 & $*$ & 8.93 & 46 & -18 & 8 & Right Heschl's gyrus (TE1.0, TE1.1) \\
\hline & & & $*$ & 8.37 & 50 & -10 & -2 & Right superior temporal gyrus (TE1.0) \\
\hline \multicolumn{9}{|c|}{ Effects of action $\left(p_{\text {corr. }}=0.05\right.$, extent threshold $=100$ voxels $)$} \\
\hline \multirow[t]{2}{*}{1} & * & 507 & $*$ & 7.94 & 34 & -14 & 52 & Right pre-central gyrus (BA6) \\
\hline & & & * & 7.49 & 36 & -16 & 58 & Right pre-central gyrus (BA6) \\
\hline 1 & * & 42 & $*$ & 4.92 & -36 & -12 & 56 & Left pre-central gyrus (BA6) \\
\hline
\end{tabular}

$* p<0.05$ (Corrected).

\subsection{Behavioural Test Results}

Table 3 contains participant's performance (percentage of correct answers) when the pitch discrimination task is performed in quiet and in noisy background for low and high frequency category of tones described in the method section. The average values and their standard deviations are given at the bottom of the table. Due to the data that are not normally distributed, Wilcoxon signed rank test was used to compare the average values. No significant difference $(p=0.463, Z=-0.734)$ in the participants' performance is observed when they discriminate high frequency tones in both quiet and noisy background. Insignificant $(p=0.530, Z=-0.628)$ results in participants' performance were also found in discriminating low frequency tones both in quiet and in noise. However, participants showed a significantly better performance in discriminating low frequency tones as compared to high frequency tones when the task was performed either in quiet $(p<0.05, Z=-2.760)$ or in noisy background $(p<0.05, Z=-2.760)$. These results indicate that the participants' performance in discriminating between $f_{\mathrm{o}}$ and $f_{4}$ is largely influenced by the tonal frequencies from which their performance is better in discriminating between $f_{\mathrm{o}}$ and $f_{4}$ of low-frequency category as compared to that of high-frequency category. The influence of background noise on the participants' performance is however minimal.

Table 3. Behavior analysis results obtained based on individual participant's number of correct answers

\begin{tabular}{cccccc}
\hline & \multicolumn{2}{c}{$\begin{array}{c}\text { Correct answers for } \\
\text { low Frequency tones (\%) }\end{array}$} & & \multicolumn{2}{c}{$\begin{array}{c}\text { Correct answers for } \\
\text { High frequency tones (\%) }\end{array}$} \\
\cline { 2 - 3 } \cline { 5 - 6 } Participants & In quiet & In noise & & In quiet & In noise \\
\hline S1 & 84.62 & 88.57 & & 81.82 & 75.68 \\
S2 & 84.62 & 80.00 & & 72.73 & 72.97 \\
S3 & 53.85 & 54.29 & & 45.45 & 59.46 \\
S4 & 56.41 & 54.29 & & 54.55 & 51.35 \\
S5 & 94.87 & 85.71 & & 87.88 & 81.08 \\
S6 & 92.31 & 88.57 & & 87.88 & 78.38 \\
S7 & 82.10 & 80.00 & & 81.82 & 78.38 \\
S8 & 76.92 & 71.43 & & 66.67 & 64.87 \\
S9 & 97.44 & 94.29 & & 78.79 & 81.08 \\
S10 & 46.15 & 60.00 & & 51.52 & 48.65 \\
S11 & 69.23 & 65.71 & & 54.55 & 59.46 \\
S12 & 58.97 & 77.14 & & 57.58 & 78.38 \\
S13 & 100.00 & 100.00 & & 93.94 & 78.38 \\
Average & $76.73(18.12)$ & $76.92(14.91)$ & & $70.40(16.23)$ & $69.86(11.62)$ \\
\hline
\end{tabular}




\subsection{The Differential Effects of Background Noise and Tonal Frequencies}

Differential effects of performing pitch discrimination task in quiet and in noise using low and high frequency tones were investigated using paired $t$-test in the second level analysis. At a corrected significance level of $\alpha=$ 0.05 , not a single voxel or cluster of voxels is activated in the comparison when the participants i) discriminate $f_{\mathrm{o}}$ and $f_{4}$ of low-frequency category between in noise and in quiet, ii) discriminate $f_{\mathrm{o}}$ and $f_{4}$ of high-frequency category between in noise and in quiet, iii) discriminate $f_{0}$ and $f_{4}$ between those of low-frequency category and high-frequency category in quiet and iv) discriminate $f_{\mathrm{o}}$ and $f_{4}$ between those of low-frequency category and high-frequency category in noise; with distracting tones of frequencies $f_{1}, f_{2}$ and $f_{3}$ being given in between $f_{\mathrm{o}}$ and $f_{4}$. Thus, a much lower significance level (uncorrected $\alpha=0.001$ ) with an extent threshold of 100 voxels was used for further analysis.

At a lower threshold, when the participants discriminated $f_{\mathrm{o}}$ and $f_{4}$ of low-frequency category, there is no voxels that are significantly more active when the task is performed in quiet than in noise but there are voxels that are significantly more active when the task is performed in noise as compared to in quiet. The results are shown in Table 4. It can be seen from the table that voxels that are significantly more active when the task is performed in noisy as compared to in quiet background during low-frequency tone discrimination are mainly located in the bilateral primary auditory areas specifically in the bilateral superior temporal gyrus (TE1.0, TE1.1 and TE1.2), left Heschl's gyrus (TE1.1) and part of insula lobe. A larger number of activated voxels and higher $t_{\mathrm{df}}$ values can be observed for the right hemispheric regions.

In contrast, when the participants discriminated $f_{\mathrm{o}}$ and $f_{4}$ of high-frequency category, no voxels are significantly more active when the task is performed in noise than in quiet but there are voxels that are significantly more active when the task is performed in quiet as compared to in noise. It can be seen from Table 4 that voxels that are significantly more active when the task is performed in quiet as compared to in noisy background during high-frequency tone discrimination are totally different from the previous results. These voxels are mainly located in the bilateral putamen and left hippocampus with a larger number of activated voxels and higher $t$ values for the right hemispheric region.

When the participants discriminated between $f_{\mathrm{o}}$ and $f_{4}$ in quiet background, there are voxels that are significantly more active when the task is performed using low-frequency tones as compared to high-frequency tones. These voxels are located in Heschl's gyrus (TE1.0 and TE1.1). In contrast, voxels that are significantly more active when the task is performed using high-frequency tones as compared to low-frequency tones fall in the area of left middle temporal gyrus and left angular gyrus in the left inferior parietal lobe (Table 5). In noisy condition, when the participants discriminated between $f_{\mathrm{o}}$ and $f_{4}$, voxels that are significantly more active when the task is performed using low-frequency tones as compared to high-frequency tones are in the areas of bilateral superior temporal gyrus and Heschl's gyrus (TE1.0, TE1.1 and TE1.2) with a larger number of activated voxels but with lower $t$ values for the right hemispheric regions. In the same noisy condition, no voxels are significantly more active when the task is performed using high-frequency tones as compared to low-frequency tones (Table 5).

\section{Discussion}

For listening only to noise ( $N$ condition), both the group RFX and FFX results indicate bilateral primary auditory cortex activation centered in the right Heschl's gyrus. A higher number of activated voxels in the right primary auditory cortex is accompanied by a higher $t$ statistics at the point (voxel) of maximum intensity and number of activated voxels as compared to the left primary auditory cortex. Not only did the number of activated voxels and the $t$ statistics for the primary auditory cortex in the two hemispheres differ in values, the magnitude of the difference was also considerable. Based on previous findings (Hwang et al., 2005), more voxels have been found to be activated in the right temporal lobe as compared to the left when noise is used as stimulus, due its (right temporal lobe) higher sensitivity to non-speech stimulus such as pure tones and noise. Speech stimulus, on the other hand, will most likely activate the left temporal lobe relatively higher than the right temporal lobe especially if the perception of words comes into play and only if the stimulus task is linguistically demanding (Hwang et al., 2005). Similar average temporal lobe activation have also been found in our previous study using binaurally delivered white noise stimulus on five right handed male participants (Yusoff et al., 2011) and ten right handed mixed participants (Abdul Hamid et al., 2012).

Performing pitch discrimination task in quiet (SQY and SQN) and in noisy (SNY and SNN) conditions have evoked responses in many cortical areas as revealed by conjunction analysis (Friston et al., 1999) results shown in Table 1 and Figure 2. On the statistical parametric maps (not shown), the spatial activation obtained for each of SQY, SQN, SNY and SNN condition shows little difference from each other. Our interpretation was that the brain executed a similar network during the execution of the pitch discrimination task even though it was 
conducted in four different conditions. This could be due to the fact that the same regions in the brain responded to the pitch discrimination task used in this study either it was performed in quiet or in noise or either the participants press or did not press the squeeze bulb at the end of the task. Based on the areas of activation, the network executed by the brain under the pitch discrimination task clearly involves sensory, perceptual and cognitive functions. The results obtained from detailed analyses using paired- $t$ test performed between conditions, however, show significant difference in the activation extent between performing the task in quiet and in noise and between pressing and not pressing the squeeze bulb. This will be further discussed in the following section.

The activated brain areas that are common to all conditions during the performance of the pitch discrimination task, as shown in Figure 2, have been found to be activated in many previous fMRI studies that used auditory stimulus. In a study by Gaab et al. (2003), supplementary motor area, superior temporal gyrus, cerebellum, inferior frontal gyrus and inferior frontal lobes were found to be activated when the participants performed pure tone discrimination. However, the activation in the insula, putamen and precentral gyrus was absent in their study. These regions could have been detected if a lower height threshold was used. In another interesting work by the same author (Gaab \& Schlaug, 2003), pitch discrimination task performance was compared between musicians and non-musicians. Bilateral activation was observed in the superior temporal gyrus, supramarginal gyrus, posterior middle frontal gyrus, inferior frontal gyrus and superior parietal lobe. Musicians were found to perform better in pitch discrimination task than non-musician with significantly higher activation in the right temporal and supramarginal gyrus, while non-musician rely more on the right primary and left secondary auditory cortex in executing the task.

Table 4. Brain activation characteristics obtained from paired $t$-test in the second level analysis to obtain the differential effects of performing frequency discrimination task in quiet and in noise using low and high frequency tones

\begin{tabular}{|c|c|c|c|c|c|c|c|c|}
\hline \multirow{2}{*}{$\begin{array}{l}\text { No. of } \\
\text { active } \\
\text { cluster }\end{array}$} & \multirow{2}{*}{$\begin{array}{c}\text { Cluster } \\
\text { level } \\
p \text {-value }\end{array}$} & \multirow{2}{*}{$\begin{array}{l}\text { No. of } \\
\text { active } \\
\text { voxels }\end{array}$} & \multirow{2}{*}{$\begin{array}{c}\text { Peak } \\
\text { level } \\
p \text {-value }\end{array}$} & \multirow{2}{*}{$\begin{array}{l}\text { Peak level } \\
t_{12} \text { value }\end{array}$} & \multicolumn{3}{|c|}{ MNI coordinates } & \multirow[t]{2}{*}{ Activated areas } \\
\hline & & & & & $x$ & $y$ & $z$ & \\
\hline \multicolumn{9}{|c|}{ Discriminating low frequency tones: in Quiet $>$ in Noise $\left(p_{\text {uncorr. }}=0.001\right.$, extent threshold $=100$ voxels $)$} \\
\hline \multicolumn{9}{|c|}{-No significant activation cluster - } \\
\hline \multicolumn{9}{|c|}{ Discriminating low frequency tones: in Noise $>$ in Quiet $\left(p_{\text {uncorr. }}=0.001\right.$, extent threshold $=100$ voxels) } \\
\hline \multirow[t]{6}{*}{2} & \multirow[t]{3}{*}{$*$} & \multirow[t]{3}{*}{357} & $*$ & 8.56 & 58 & -16 & 6 & Right superior temporal gyrus \\
\hline & & & $*$ & 6.93 & 48 & -10 & 4 & Right insula lobe \\
\hline & & & * & 5.33 & 62 & 0 & -2 & Right superior temporal gyrus \\
\hline & \multirow[t]{3}{*}{ * } & \multirow[t]{3}{*}{241} & $*$ & 7.56 & -50 & -14 & 2 & Left superior temporal gyrus \\
\hline & & & $*$ & 6.34 & -42 & -22 & 4 & Left superior temporal gyrus \\
\hline & & & $*$ & 5.69 & -36 & -28 & 8 & Left Heschl's gyrus \\
\hline \multicolumn{9}{|c|}{ Discriminating high frequency tones: in Quiet $>$ in Noise ( $p_{\text {uncorr. }}=0.001$, extent threshold $=100$ voxels) } \\
\hline \multirow[t]{5}{*}{2} & \multirow[t]{2}{*}{0.001} & \multirow[t]{2}{*}{150} & $*$ & 6.78 & 30 & -10 & 4 & Right putamen \\
\hline & & & * & 6.68 & 36 & 4 & -4 & - \\
\hline & \multirow[t]{3}{*}{0.004} & \multirow[t]{3}{*}{103} & * & 5.63 & -32 & -10 & 0 & Left putamen \\
\hline & & & * & 4.49 & -30 & 8 & -4 & Left putamen \\
\hline & & & 0.001 & 4.24 & -34 & -16 & -12 & Left hippocampus \\
\hline \multicolumn{9}{|c|}{ Discriminating high frequency tones: in Noise $>$ in Quiet $\left(p_{\text {uncorr. }}=0.001\right.$, extent threshold $=100$ voxels) } \\
\hline \multicolumn{9}{|c|}{-No significant activation cluster - } \\
\hline
\end{tabular}


Table 5. Brain activation characteristics obtained from paired t-test in the second level analysis to obtain the differential effects of discriminating between low and high frequency tones in quiet and in noise

\begin{tabular}{|c|c|c|c|c|c|c|c|c|}
\hline \multirow{2}{*}{$\begin{array}{l}\text { No. of } \\
\text { active } \\
\text { cluster }\end{array}$} & \multirow{2}{*}{$\begin{array}{c}\text { Cluster } \\
\text { level } \\
p \text {-value }\end{array}$} & \multirow{2}{*}{$\begin{array}{l}\text { No. of } \\
\text { active } \\
\text { voxels }\end{array}$} & \multirow{2}{*}{$\begin{array}{l}\text { Peak level } \\
p \text {-value }\end{array}$} & \multirow{2}{*}{$\begin{array}{c}\text { Peak level } \\
t_{12} \text { value }\end{array}$} & \multicolumn{3}{|c|}{ MNI coordinates } & \multirow[t]{2}{*}{ Activated areas } \\
\hline & & & & & $x$ & $y$ & $z$ & \\
\hline \multicolumn{9}{|c|}{ Discriminating in quiet: Low frequency $>$ High frequency $\left(p_{\text {uncorr. }}=0.001\right.$, extent threshold $=100$ voxels $)$} \\
\hline 1 & 0.001 & 143 & $*$ & 6.3 & 44 & -24 & 10 & Right Heschl's gyrus \\
\hline \multicolumn{9}{|c|}{ Discriminating in quiet: High frequency $>$ Low frequency $\left(p_{\text {uncorr. }}=0.001\right.$, extent threshold $=100$ voxels) } \\
\hline \multirow[t]{2}{*}{1} & 0.003 & 119 & $*$ & 6.42 & -52 & -62 & 16 & Left middle temporal gyrus \\
\hline & & & $*$ & 5.46 & -58 & -64 & 24 & Left angular gyrus \\
\hline \multicolumn{9}{|c|}{ Discriminating in noise: Low frequency $>$ High frequency $\left(p_{\text {uncorr. }}=0.001\right.$, extent threshold $=100$ voxels $)$} \\
\hline \multirow[t]{6}{*}{2} & $*$ & 309 & $*$ & 7.3 & -52 & 2 & -8 & Left superior temporal gyrus \\
\hline & & & $*$ & 6.23 & -48 & -16 & 0 & Left superior temporal gyrus \\
\hline & & & $*$ & 5.31 & -36 & -26 & 0 & - \\
\hline & $*$ & 353 & $*$ & 6.49 & 58 & -16 & 10 & $\begin{array}{l}\text { Right superior temporal } \\
\text { gyrus }\end{array}$ \\
\hline & & & $*$ & 5.86 & 42 & -24 & 10 & Right Heschl's gyrus \\
\hline & & & $*$ & 4.64 & 42 & -18 & 2 & $\begin{array}{l}\text { Right superior temporal } \\
\text { gyrus }\end{array}$ \\
\hline \multicolumn{9}{|c|}{ Discriminating in noise: High frequency $>$ Low frequency $\left(p_{\text {uncorr. }}=0.001\right.$, extent threshold $=100$ voxels $)$} \\
\hline \multicolumn{9}{|c|}{-No significant activation cluster - } \\
\hline
\end{tabular}

As mentioned above, the pitch discrimination task used in this study involves sensory, perceptual and cognitive strategies which are listening to the delivered tones, perceiving the tones with attention, recognizing the tones and finally making decision (or to discriminate) whether the fourth or final tone $\left(f_{4}\right)$ in a series of distracting tones is similar or not similar to the target tone $\left(f_{\mathrm{o}}\right)$. The involvement of bilateral primary auditory cortex, in particular the bilateral superior temporal gyrus, as processing centers is crucial in all the four conditions. This is due to the use of auditory stimuli such as noise and pure tones, delivered binaurally through both ears (Hall \& Plack, 2009). Many previous studies using auditory stimuli that were delivered binaurally have shown significant activation in the bilateral primary auditory regions regardless whether the participants intentionally (Gaab et al., 2003; Hwang et al., 2005; Mueller et al., 2011) or unintentionally (Langers \& van Dijk, 2011) listened to the stimuli. The activation of cerebellum, pre-central gyrus and supplementary motor area during the pitch discrimination task resulted from the participants' strategy in responding to the given task. The three cortical areas, beside the superior temporal gyrus, are thought to play multiple important roles during the execution of pitch discrimination task. This includes from the perception of the stimuli to decision making which was whether to squeeze or not to squeeze the rubber bulb. This argument is supported by the higher $t$ values and number of significantly activated voxels (corrected for multiple comparison) shown in Table 1 for those areas, as compared to other areas.

The pre-central gyrus, also known as BA4 or the primary motor area (M1) is one of the important areas that coordinates upper limb movements such as fingers, unimanually or bimanually (Grabowska et al., 2012; Koeneke et al., 2004a; Lutz et al., 2005). It is located at the anterior wall of the middle sulcus. The activation of bilateral pre-central gyrus may indicate the involvement of motor region contralateral and also ipsilateral to the hand that squeezed the rubber bulb. The pre-central gyrus has also been known to be involved in attention while the task is being performed. In an attentive listening event-related fMRI study (Specht \& Reul, 2003), the pre-central gyrus has been found to be bilaterally activated even though the participants did not use their motor function in responding to the task. The fact that pre-central gyrus is bilaterally activated in all conditions in this study including those in which the participants were not supposed to press the bulb supports its perceptual role 
such as attention and decision making in addition to its motor role in preparing and executing the action (squeezing the rubber bulb).

The execution of pitch discrimation task in previous studies has also been found to activate the SMA (Gaab et al., 2003) and cerebellum (Gaab et al., 2003; Gaab \& Schlaug, 2003). The SMA is located in the premotor cortex or BA6. It extends anteriorly from the primary motor cortex near the Sylvian fissure (lateral sulcus). The basic function of this brain area, in which the SMA is a portion of it, is motor sequencing and movement planning. SMA is known to be the centre for movement initiation (Koeneke et al., 2004a). SMA is also known to have the function of retrieval of learned sequences that are under internal control such as the performance of a sequence of movements from memory (Lutz et al., 2005). The fact that SMA exhibits the highest activation intensity in all tone discriminating conditions is possibly related to its diversed functions with regard to tone discriminating task used in this study (Specht \& Reul, 2003). This would include the perceptual part of the pitch memory processes as well as the action (or motor) part after a decision has been made whether to press or not to press the squeeze bulb.

In the execution of motor function, cerebellum is the only brain structure outside the cortex that is involved, coordinating the movement with precision and timing (Koeneke et al., 2004b; Lutz et al., 2005). However, it does not involve in the initiation of the movement which is known to be triggered in BA4 and BA6. The activation of cerebellum in this study is in accordance with the use of participant's hand in responding to the task The result also supports earlier understanding that cerebellum would also be involved in non-motor functions in addition to its well known motor function. For the non-motor role, it may also be involved in some cognitive functions such as attention and language (Wolf et al., 2009). The activation of cerebellum (VI) bilaterally in this study is also in good agreement with previous work on pitch discrimination (Gaab et al., 2003; Gaab \& Schlaug, 2003; Petacchi et al., 2011). In a positron emission tomography (PET) study on the activation of human cerebellum during pitch discrimination (Petacchi et al., 2011), passive listening was found to elicit cerebellar activity in lobule IX while pitch discrimination increased cerebellar activity significantly in crus I and II, lobules VI, VIIB and VIIIB as compared to passive listening. The increase in brain activation was later found to be correlated with the difficulty of the discrimination task which involved lobules V, VI and IX. The results obtained from this study also complement recent findings that show pitch discrimination deficits in patients with global cerebellar degeneration who were in a great difficulty accomplishing pitch discrimination task (Parsons et al., 2009). In our recent work on verbal working memory via backward repeat test (BRT), this brain structure has been found to be involved in attenuating noise and/or increasing attention to task performance (Abdul Manan et al., 2012). Nevertheless, the role of cerebellum in pitch discrimination has yet to be fully understood, given a vast number of studies conducted. Possibility ranges from facilitating pitch discrimination to sequential ordering of auditory information.

Bilateral inferior parietal lobule, putamen, insula and right inferior frontal gyrus which were activated during the pitch discrimination task used in this work would probably due to the higher cognitive components incorporated in the given task such as attention and interpretation. The inferior parietal lobule which contains the supramarginal gyrus and angular gyrus have been known to involve in auditory spatial working memory (Alain et al., 2008). The study suggested dual roles for the right inferior parietal lobule in auditory working memory, one involved in monitoring and updating sound location independent of motor response and another that underlies the integration of sensory and motor functions. In this study, the tones given in the tone discrimination task are auditory in nature and are possibly interpreted in the inferior parietal lobule during the accomplishment of the task. Putamen works in conjunction with other motor-related areas to control many types of motor skills. These include controlling motor learning, motor performance and tasks (DeLong et al., 1984a), motor preparation (Alexander \& Crutcher, 1990), specifying amplitudes of movement (Delong et al., 1984b) and movement sequences (Marchand et al., 2009; Marchand et al., 2008). It has also been hypothesized to play a role in the selection of movement and the automatic performance of previously learned movement (Marchand et al., 2009). The insula is believed to be involved in consciousness and play a role in diverse functions usually linked to emotion or the regulation of the body's homeostasis. These functions include perception, motor control, self-awareness, cognitive functioning, and interpersonal experience (Karnath et al., 2005). The right inferior frontal gyrus has been known to be involved in go/no go tasks (Aron et al., 2004). In such a task, the participant has to inhibit a prepotent response (for instance stop pressing a button when a red signal appears) (Aron \& Poldrack, 2005) somewhat similar to what is done in the tone discrimination task in this study when the participants notice that the two tones to be discriminated are similar (to press the squeeze bulb) or not similar (not to press the squeeze bulb).

The brain activation for $N$ condition obtained earlier using RFX and FFX frameworks are the results of attentive listening in which the participants were required to listen and pay attention to the white noise delivered through 
their both ears (Abdul Hamid et al., 2010). In contrast, the activation obtained from differential brain activation analysis (either in RFX or FFX framework) is due to inattentive listening to the delivered noise that appears as a background while attending to the series of pure tone stimuli (Langers \& van Dijk, 2011). While RFX results show little hemispheric differences, a better differential brain activation results due to inattentive listening is observed when comparison is made in the FFX framework. During the tone discrimination task in noise, the participants were assumed to be in a state of unintentionally listening to the noise stimulus while trying to discriminate between the target and the fourth tone (Langers \& van Dijk, 2011). What has been obtained from the comparison, listening intentionally (during $N$ condition) and unintentionally (during tone discriminating task) to the noise stimulus exhibited marked difference in the height and spatial extents of activation in the auditory region. Attentive listening to noise stimulus activated only the Heschl's gyrus bilaterally, while inattentive listening evoked additional and wider responses in bilateral superior temporal gyrus. The activation peak or processing center changed from right hemispheric Heschl's gyrus ( $t=8.48,568$ voxels) during attentive listening to left hemispheric superior temporal gyrus $(t=10.41,1001$ voxels) during inattentive listening. This indicates the occurence of laterality shift of auditory processing, from purely sensory to that containing cognitive functions such as perceptual, attention and decision making (Hwang et al., 2005). Furthermore, as compared to attentive listening, the number of activated voxels in both hemispheres increases markedly in inattentive listening to noise during the pitch discrimination task indicating a larger auditory area that has been recruited to accomplish both the sensory and cognitive tasks.

A good agreement between FFX and RFX results can also be seen for the effects of action when the participants decided to press the squeeze bulb during the pitch discrimination task (Table 2). Dominant activation area observed in the right pre-central gyrus is due to the contralateral behavior of motor responses in the cortex as a result of the use of left fingers by the participants to press the squeeze bulb (Ahmad et al., 2011; Grabowska et al., 2012). A small ipsilateral activation in the left pre-central gyrus as indicated by the FFX results suggests the involvement of the left motor area in coordinating the action.

Behaviorally, the participants performed pitch discrimination task equally good in quiet and noisy backgrounds, either when discriminating the low or high frequency category of tones (Columns 2 vs. 3 and columns 4 vs. 5 of Table 3) with an average of $77 \%$ and $70 \%$ of their decisions in discriminating low and high frequency category of tones respectively, were correct. This behavioral test results are in good agreement with differential activation results at a corrected significant level of $\alpha=0.05$, from which not a single voxel or cluster of voxels is activated in the comparison when the participants discriminate $f_{0}$ and $f_{4}$ of low-frequency and high frequency category between in noise and in quiet. From the results obtained, it can be said that task performance seemed to be less influenced by the background. Based on the behavioral test results, one possible explanation rests on the ability of the brain to eliviate its performance in accomplishing the tone discrimination task especially when it is distracted by noise, thus equalizing the performance it has in quiet, an argument similar to the idea of stochastic resonance (Abdul Manan et al., 2012; Moss et al., 2004). However, to determine the main areas in the brain that are responsible for the compensation, a lower height threshold was implemented.

Lowering the height threshold of the SPM map to an uncorrected $p=0.001(k=100)$ revealed additional information on brain activity due to the respective tone discrimination tasks (Table 4). The absence of activated voxels for low frequency tone discrimination (in quiet $>$ in noise) and for high frequency tone discrimination (in noise $>$ in quiet) and the presence of activated voxels for low frequency tone discrimination (in noise $>$ in quiet) and for high frequency tone discrimination (in quiet $>$ in noise) suggest that low and high frequency tones have different effects on brain activation but depending on whether the task is performed in quiet or in noise. The bilateral auditory areas including the right insula lobe seemed to be more sensitive to low frequency tone discrimination but only if the task is performed in noisy condition. Likewise, putamen and hippocampus, the two brain areas that have been known to be involved in motor performance and working memory respectively (Abdul Manan et al., 2012), showed significantly higher activation in quiet as compared to in noise because they are more sensitive to high frequency tones discrimination but only when the task is performed in quiet. In conjunction with the behavioral test results explained earlier, one possible explanation for the significant increase in the brain activation in the bilateral auditory areas for the respective (Noise > Quiet) condition mentioned above is to compensate for the degradation of cognitive processing performance that has been affected by the white noise which distract attention from the main tasks. In our previous study on the effects of noise on verbal working memory processing, the bilateral primary auditory cortices have shown significant increase in activation in noisy condition as compared to in quiet (Abdul Manan et al., 2012). In the case of (Quiet > Noise) condition, comparison with behavioral test results is not possible due its one-way nature of test. 
Based on the behavior test results, low frequency tones have been found to be more intelligible to be discriminated as compared to high frequency tones regardless of whether the task is done in quiet or in noisy background. Based on the differential brain activation results at $p=0.001$ ( $k=100$ voxels) shown in Table 5 , low frequency tonal intelligibility (low frequency $>$ high frequency) can be associated with the enhancement of activation in the bilateral primary auditory cortex which are the right Heschl's gyrus for the discrimination task done in quiet and bilateral superior temporal gyrus and right Heschl's gyrus for the discrimination task done in noisy condition. In contrast, high frequency tonal intelligibility is in accordance with significant differential brain activation in the left middle temporal gyrus and left angular gyrus which appears only when the discrimination task is done in quiet (high frequency $>$ low frequency).

There are several limitations in the study design that may have adversely affected the results. The first concern is about the potential habituation of each participant. Varied individual personality, comfort-level in the scanner and exposure to past scans could potentially introduce additional physiological noise into the fMRI results, affecting habituation and leading to erroneous conclusions. Possible solutions to habituation would be prior and repeated exposure to the entire experimental paradigm (including sensations and sounds) in a mock-scanner setup to at least minimize the "initial novelty" of this experience. In addition, the participants could be put through an initial period of exposure at the beginning of the experiment to ensure habituation prior to recording actual data. The second concern is about the duration of dead time during which the participant's brain is supposedly recovering from the scanner sound. This rest period of no stimulus delivery which is in between the EPI scan and the next stimulus presentation should be made longer for a complete recovery of hemodynamic response from the scanner sound. The third concern is about the number of participants that need to be added in the future study to achieve sufficient power and specificity which in turn would allow inference to be made at corrected significant level.

\section{Conclusion}

The pitch discrimination task implemented in this study has been able to elucidate several important information with regard to pitch memory processing. Human brain appears to execute auditory pitch memory processing by activating multiple areas that are associated with listening, attending, recognizing, perceptual and decision making. Similar integrative network consisting of bilateral superior temporal gyrus, pre-central gyrus, cerebellum, insula, putamen, inferior parietal lobe and supplementary motor area is established in the brain for pitch discrimination task performed in quiet (with and without participants respond) and pitch discrimination task performed in noisy background (with and without participants respond). Further analysis conducted on the data reveals that the bilateral primary auditory area is enhanced when the pitch discrimination task is performed in noisy background so that the performance equals to that in quiet background as suggested by behavioral study results. This sensory area has also been found to play important roles in facilitating low frequency discrimination in both quiet and noisy backgrounds. Low and high frequency tones have different effects on pitch memory processing which in turn affects the brain activation depending on whether the task is performed in quiet or in noise. Significant activation in the primary auditory and motor area discussed above validates the tone discrimination task used in this study. The results also highlighted the importance of using both group FFX and RFX analyses for comparison in making inference about the brain activation based on anatomically closed hypothesis, and especially when the number of participants is relatively small.

\section{Acknowledgements}

The authors would like to thank Sa'don Samian, the MRI Technologist of the Universiti Kebangsaan Malaysia Medical Centre (UKMMC), for the assistance in the scanning, the Department of Radiology, Universiti Kebangsaan Malaysia Hospital for the permission to use the MRI scanner and Audiology Program, Faculty of Health Sciences UKM for conducting hearing test on the participants. This work was supported by the research grant eScience Fund 06-01-02-SF0548, the Ministry of Science, Technology and Innovation of Malaysia.

\section{References}

Abdul Hamid, K., Yusoff, A. N, Abdul Rahman, M. Z., Mohamad, M., \& Abd Hamid, A. I. (2012). Effective connectivity between superior temporal gyrus and Heschl's gyrus during white noise listening: linear versus non-linear models. Biomedical Imaging and Intervention Journal, 8(2), e13.

Abdul Hamid, K., Yusoff, A. N., Mohamad, M., Abd Hamid, A. I., \& Abdul Manan, H. (2010). Comparing Intrinsic Connectivity Models for the Primary Auditory Cortices. Progress of Physics Research in Malaysia, Perfik 2009, 1250, 389-392. 
Abdul Manan, H., Franz, E. A., Yusoff, A. N., \& Mukari, S. Z. M. (2012). Hippocampal-Cerebellar involvement in enhancement of performance in word-based BRT with the presence of background noise: an initial fMRI study. Psychology and Neuroscience, 5(2), 247-256. http://dx.doi.org/10.3922/j.psns.2012.2.16

Ahmad, N. Z., Ismafairus, A. H. A., Khairiah, A. H., Kamil, W. W. A., Mazlyfarina, M., \& Hanani, A. M. (2011). Functional specialisation and effective connectivity during self-paced unimanual and bimanual tapping of hand fingers: An extended analysis using dynamic causal modeling and Bayesian model selection for group studies. Malaysian Journal of Medicine and Health Sciences, 7(2), 17-36.

Alain, C., He, Y., \& Grady, C. (2008). The contribution of the inferior parietal lobe to auditory spatial working memory. Journal of Cognitive Neuroscience, 20(2), 285-295. http://dx.doi.org/10.1162/jocn.2008.20014

Alexander, G. E., \& Crutcher, M. D. (1990). Preparation for movement: neural representations of intended direction in three motor areas of the monkey. Journal of Neurophysiology, 64(1), 133-150.

Aron, A. R., \& Poldrack, R. A. (2005). The cognitive neuroscience of response inhibition: relevance for genetic research in attention-deficit/hyperactivity disorder. Biological Psychiatry, 57(11), 1285-1292.

Aron, A. R., Robbins, T. W., \& Poldrack, R. A. (2004). Inhibition and the right inferior frontal cortex. Trends Cogn Sci, 8(4), 170-177. http://dx.doi.org/10.1016/j.tics.2004.02.010

Czachowska-Sieszycka, B., \& Budohoska, W. (1979). The effect of distractor on memory for pitch: trace distortion or interference with retrieval? Acta Neurobiol Exp (Wars), 39(1), 15-25.

DeLong, M. R., Alexander, G. E., Georgopoulos, A. P., Crutcher, M. D., Mitchell, S. J., \& Richardson, R. T. (1984a). Role of basal ganglia in limb movements. Human Neurobiology, 2(4), 235-244.

DeLong, M. R., Georgopoulos, A. P., Crutcher, M. D., Mitchell, S. J., Richardson, R. T., \& Alexander, G. E. (1984b). Functional organization of the basal ganglia: contributions of single-cell recording studies. Ciba Foundation Symposium, 107, 64-82.

Deutsch, D. (1970). Tones and numbers: specificity of interference in immediate memory. Science, 168(3939), 1604-1605. http://dx.doi.org/10.1126/science.168.3939.1604

Deutsch, D. (1972a). Effect of repetition of standard and comparison tones on recognition memory for pitch. Journal of Experimental Psychology, 93(1), 156-162. http://dx.doi.org/10.1037/h0032496

Deutsch, D. (1972b). Mapping of interactions in the pitch memory store. Science, 175(4025), 1020-1022. http://dx.doi.org/10.1126/science.175.4025.1020

Deutsch, D. (1978). Pitch memory: an advantage for the left-handed. Science, 199(4328), 559-560. http://dx.doi.org/10.1126/science.622558

Eickhoff, S. B., Stephan, K. E., Mohlberg, H., Grefkes, C., Fink, G. R., Amunts, K., \& Zilles, K. (2005). A new SPM toolbox for combining probabilistic cytoarchitectonic maps and functional imaging data. Neuroimage, 25(4), 1325-1335. http://dx.doi.org/10.1016/j.neuroimage.2004.12.034

Friston, K. J., Frith, C. D., Liddle, P. F., \& Frackowiak, R. S. J. (1991). Comparing functional (PET) images: the assessment of significant change. Journal of Cerebral Blood Flow and Metabolisme, 11(4), 690-699. http://dx.doi.org/10.1038/jcbfm.1991.122

Friston, K. J. (2005). Models of brain function in neuroimaging. Annual Review of Psychology, 56, 57-87. http://dx.doi.org/10.1146/annurev.psych.56.091103.070311

Friston, K. J., Holmes, A., Poline, J. B., Price, C. J., \& Frith, C. D. (1996). Detecting activations in PET and fMRI: levels of inference and power. Neuroimage, 4(3), 223-235. http://dx.doi.org/10.1006/nimg.1996.0074

Friston, K. J., Holmes, A. P., Price, C. J., Buchel, C., \& Worsley, K. J. (1999). Multisubject fMRI studies and conjunction analyses. Neuroimage, 10(4), 385-396. http://dx.doi.org/10.1006/nimg.1999.0484

Gaab, N., Gaser, C., Zaehle, T., Jancke, L., \& Schlaug, G. (2003). Functional anatomy of pitch memory - an fMRI study with sparse temporal sampling. Neuroimage, 19(4), 1417-1426. http://dx.doi.org/10.1016/S1053-8119(03)00224-6

Gaab, N., \& Schlaug, G. (2003). The effect of musicianship on pitch memory in performance matched groups. Neuroreport, 14(18), 2291-2295. http://dx.doi.org/10.1097/00001756-200312190-00001 
Grabowska, A., Gut, M., Binder, M., Forsberg, L., Rymarczyk, K., \& Urbanik, A. (2012). Switching handedness: fMRI study of hand motor control in right-handers, left-handers and converted left-handers. Acta Neurobiol Exp (Wars), 72(4), 439-451.

Hall, D. A., \& Plack, C. J. (2009). Pitch processing sites in the human auditory brain. Cerebral Cortex, 19(3), 576-585. http://dx.doi.org/10.1093/cercor/bhn108

Hwang, J. H., Wu, C. W., Chou, P. H., Liu, T. C., \& Chen, J. H. (2005). Hemispheric difference in activation patterns of human auditory-associated cortex: An fMRI study. Orl-Journal for Oto-Rhino-Laryngology and Its Related Specialties, 67(4), 242-246. http://dx.doi.org/10.1159/000089501

Karnath, H. O., Baier, B., \& Nagele, T. (2005). Awareness of the functioning of one's own limbs mediated by the insular cortex? Journal of Neuroscience, 25(31), 7134-7138.

Koeneke, S., Lutz, K., Wustenberg, T., \& Jancke, L. (2004a). Bimanual versus unimanual coordination: what makes the difference? Neuroimage, 22(3), 1336-1350. http://dx.doi.org/10.1016/j.neuroimage.2004.03.012

Koeneke, S., Lutz, K., Wustenberg, T., \& Jancke, L. (2004b). Long-term training affects cerebellar processing in skilled keyboard players. Neuroreport, 15(8), 1279-1282.

Langers, D. R., \& van Dijk, P. (2011). Mapping the Tonotopic Organization in Human Auditory Cortex with Minimally Salient Acoustic Stimulation. Cerebral Cortex.

Langers, D. R., van Dijk, P., \& Backes, W. H. (2005). Lateralization, connectivity and plasticity in the human central auditory system. Neuroimage, 28(2), 490-499. http://dx.doi.org/10.1016/j.neuroimage.2005.06.024

Lutz, K., Koeneke, S., Wustenberg, T., \& Jancke, L. (2005). Asymmetry of cortical activation during maximum and convenient tapping speed. Neuroscience Letters, 373(1), 61-66. http://dx.doi.org/10.1016/j.neulet.2004.09.058

Marchand, W. R., Lee, J. N., Healy, L., Thatcher, J. W., Rashkin, E., Starr, J., \& Hsu, E. (2009). An fMRI motor activation paradigm demonstrates abnormalities of putamen activation in females with panic disorder. Journal of Affective Disorders, 116(1-2), 121-125. http://dx.doi.org/10.1016/j.jad.2008.10.026

Marchand, W. R., Lee, J. N., Thatcher, J. W., Hsu, E. W., Rashkin, E., Suchy, Y., ... Barbera, S. S. (2008). Putamen coactivation during motor task execution. Neuroreport, 19(9), 957-960. http://dx.doi.org/10.1097/WNR.0b013e328302c873

Moore, R. E., Estis, J., Gordon-Hickey, S., \& Watts, C. (2008). Pitch discrimination and pitch matching abilities with vocal and nonvocal stimuli. Journal of Voice, 22(4), 399-407. http://dx.doi.org/10.1016/j.jvoice.2006.10.013

Moore, R. E., Keaton, C., \& Watts, T. (2007). The role of pitch memory in pitch discrimination and pitch matching. Journal of Voice, 21(5), 560-567. http://dx.doi.org/10.1016/j.jvoice.2006.04.004

Moss, F., Ward, L. M., \& Sannita, W. G. (2004). Stochastic resonance and sensory information processing: a tutorial and review of application. Clinical Neurophysiology, 115(2), 267-281. http://dx.doi.org/10.1016/j.clinph.2003.09.014

Mueller, K., Mildner, T., Fritz, T., Lepsien, J., Schwarzbauer, C., Schroeter, M. L., \& Moller, H. E. (2011). Investigating brain response to music: a comparison of different fMRI acquisition schemes. Neuroimage, 54(1), 337-343. http://dx.doi.org/10.1016/j.neuroimage.2010.08.029

Novitski, N., Tervaniemi, M., Huotilainen, M., \& Naatanen, R. (2004). Frequency discrimination at different frequency levels as indexed by electrophysiological and behavioral measures. Brain Research. Cognitive Brain Research, 20(1), 26-36. http://dx.doi.org/10.1016/j.cogbrainres.2003.12.011

Ogawa, S., Lee, T. M., Nayak, A. S., \& Glynn, P. (1990). Oxygenation-sensitive contrast in magnetic resonance image of rodent brain at high magnetic fields. Magnetic Resonance in Medicine, 14(1), 68-78. http://dx.doi.org/10.1002/mrm.1910140108

Oldfield, R. C. (1971). The assessment and analysis of handedness: the Edinburgh inventory. Neuropsychologia, 9(1), 97-113. http://dx.doi.org/10.1016/0028-3932(71)90067-4

Parsons, L. M., Petacchi, A., Schmahmann, J. D., \& Bower, J. M. (2009). Pitch discrimination in cerebellar patients: evidence for a sensory deficit. Brain Research, 1303, 84-96. http://dx.doi.org/10.1016/j.brainres.2009.09.052 
Petacchi, A., Kaernbach, C., Ratnam, R., \& Bower, J. M. (2011). Increased activation of the human cerebellum during pitch discrimination: a positron emission tomography (PET) study. Hearing Research, 282(1-2), 35-48. http://dx.doi.org/10.1016/j.heares.2011.09.008

Specht, K., \& Reul, J. (2003). Functional segregation of the temporal lobes into highly differentiated subsystems for auditory perception: an auditory rapid event-related fMRI-task. Neuroimage, 20(4), 1944-1954. http://dx.doi.org/10.1016/j.neuroimage.2003.07.034

Stoessel, C., Stiller, J., Bleich, S., Boensch, D., Doerfler, A., Garcia, M., ... Forster, C. (2011). Differences and similarities on neuronal activities of people being happily and unhappily in love: a functional magnetic resonance imaging study. Neuropsychobiology, 64(1), 52-60. http://dx.doi.org/10.1159/000325076

Wolf, U., Rapoport, M. J., \& Schweizer, T. A. (2009). Evaluating the affective component of the cerebellar cognitive affective syndrome. Journal of Neuropsychiatry and Clinical Neurosciences, 21(3), $245-253$. http://dx.doi.org/10.1176/appi.neuropsych.21.3.245

Yusoff, A. N., Mohamad, M., Hamid, K. A., Abd Hamid, A. I., \& Mukari, S. Z. M. S. (2011). Acquisition, Analyses and Interpretation of fMRI Data: A Study on the Effective Connectivity in Human Primary Auditory Cortices. Sains Malaysiana, 40(6), 665-678.

\section{Copyrights}

Copyright for this article is retained by the author(s), with first publication rights granted to the journal.

This is an open-access article distributed under the terms and conditions of the Creative Commons Attribution license (http://creativecommons.org/licenses/by/3.0/). 